\title{
BMJ Open Comparative safety of bowel cleansers: protocol for a systematic review and network meta-analysis
}

\author{
Pauline Barbeau, ${ }^{1}$ Dianna Wolfe, ${ }^{1}$ Fatemeh Yazdi, ${ }^{1}$ Danielle B Rice, ${ }^{1,2}$ \\ Catherine Dube, ${ }^{1,3,4}$ Salmaan Kanji, ${ }^{1,4,5}$ Alaa Rostom, ${ }^{1,3,4}$ Becky Skidmore, ${ }^{1}$ \\ David Moher, ${ }^{1,6}$ Brian Hutton ${ }^{1,6}$
}

To cite: Barbeau P, Wolfe D, Yazdi F, et al. Comparative safety of bowel cleansers: protocol for a systematic review and network meta-analysis. BMJ Open 2018:8:e021892. doi:10.1136/ bmjopen-2018-021892

- Prepublication history and additional material for this paper are available online. To view these files, please visit the journal online (http://dx.doi. org/10.1136/bmjopen-2018021892).

Received 2 February 2018

Revised 24 April 2018

Accepted 4 May 2018

Check for updates

${ }^{1}$ Clinical Epidemiology Program, Ottawa Hospital Research Institute, Ottawa, Ontario, Canada

${ }^{2}$ Department of Clinical Psychology, McGill University, Montreal, Quebec, Canada ${ }^{3}$ Department of Medicine, Division of Gastroenterology, The Ottawa Hospital, Ottawa, Ontario, Canada

${ }^{4}$ Faculty of Medicine, University of Ottawa, Ottawa, Ontario,

Canada

${ }^{5}$ Department of Pharmacy,

The Ottawa Hospital, Ottawa,

Ontario, Canada

${ }^{6}$ School of Epidemiology and

Public Health, University of

Ottawa, Ottawa, Ontario, Canada

Correspondence to

Dr Brian Hutton;

bhutton@ohri.ca

\section{ABSTRACT}

Introduction The US Food and Drug Administration has withdrawn the bowel cleansing kit HalfLytely (PEG 3500) with $10 \mathrm{mg}$ bisacodyl tablets due to an increased risk of ischaemic colitis compared with the same kit with only $5 \mathrm{mg}$ bisacodyl. This is of interest in Canada given that the bowel cleansing kit Bi-Peglyte (PEG 3500) with $15 \mathrm{mg}$ bisacodyl is currently approved for use. The objective is to assess the comparative safety of various bowel cleansers with or without bisacodyl, with a primary interest inpolyethylene glycol (PEG)-based and sodium-picosulfatebased products.

Methods and analysis Given the existing volume of the literature, the review will be conducted in two stages. Stage 1 will consist of a scoping exercise by searching MEDLINE, Embase and the Cochrane Library (up to 21 November 2017) to identify randomised controlled trials, quasirandomised studies and non-randomised studies in which any bowel cleanser regimens were compared among persons undergoing colonoscopy. The outcomes will be mapped to establish a listing of the studies and their comparisons and outcomes currently available in the literature. From this, a data synthesis plan will be determined. In stage 2, a systematic review with metaanalyses will be pursued, focused on the bowel cleanser comparisons and outcomes of interest identified in stage 1. Two reviewers will screen, extract and quality assess the articles. Outcomes of interest include ischaemic colitis, electrolyte imbalances and their consequences, seizures, bowel perforation and patient tolerability. If sufficient data exist and studies are of sufficient homogeneity, network meta-analyses (NMAs) will be performed.

Ethics and dissemination Ethics approval was not necessary due to study design. Updating the safety profile of bowel cleansers among the generally healthy population undergoing colonoscopy is pertinent given recent approval changes. This will be the first NMA within this population. Policy considerations may be reconsidered to minimise risk during bowel cleanser use.

PROSPERO registration number CRD42018084720.

\section{INTRODUCTION}

Bowel cleansers are used for various indications, the most common being preparation for colonoscopy. Prior to colonoscopy, the lower bowel must be cleared of faecal matter

\section{Strengths and limitations of this study}

- To our knowledge, this will be the first network meta-analysis focused on bowel cleanser safety in healthy colonoscopy patients.

- Although our primary focus will be on polyethylene glycol-based products with or without bisacodyl and sodium picosulfate products with or without bisacodyl, we will also consider the inclusion of other types of bowel cleansers as well.

- To determine the influence on safety, we will consider the inclusion of both randomised and non-randomised studies.

- The presence of various comorbidities may influence the type of bowel cleansers and its associated effect on outcomes; however, the availability of such data to conduct subgroup analyses may be limited.

to allow effective endoscopic visualisation of abnormalities, particularly colonic neoplasias. ${ }^{1}$ A bowel cleansing regimen is also necessary prior to gastrointestinal surgery and colonography (low-dose radiation CT) ${ }^{12}$

The safety of bowel cleansers is of importance to physicians and society as a whole given that colonoscopy is a very common procedure, which is frequently used to perform colorectal cancer screening-related indications, carried out in otherwise healthy, asymptomatic individuals. ${ }^{3}$ The safety of bowel cleansers is also important when considering the prescription of one preparation over another, especially in patients with differing comorbidities. For example, sodium phosphate $(\mathrm{NaP})$ is strictly contraindicated in patients with congestive heart failure or chronic renal disease, because it may cause phosphate nephropathy, and polyethylene glycol electrolyte lavage solution (PEG-ELS) is preferred in this population. ${ }^{4}$ Even in patients without significant comorbidities, the safety of bowel cleansers has been questioned. In 2011, the US Food and Drug Administration (FDA) withdrew 
the HalfLytely with $10 \mathrm{mg}$ bisacodyl tablets bowel preparation kit due to safety concerns. ${ }^{5}$ Compared with a kit containing $5 \mathrm{mg}$ bisacodyl, there was a greater risk of ischaemic colitis and abdominal cramping. ${ }^{5}$ In Canada, there are several approved bowel cleansers, with at least one kit (Bi-PegLyte) containing $15 \mathrm{mg}$ of bisacodyl tablets. Given the FDA's withdrawal of HalfLytely with $10 \mathrm{mg}$ bisacodyl, there is a need to review the safety of bowel cleanser kits, with or without bisacodyl in generally health patients undergoing colonoscopy.

While the efficacy of bowel cleanser preparations has been addressed in several past systematic reviews, safety endpoints are often overlooked, ${ }^{6-8}$ patient populations are often not limited to healthy patients undergoing colonoscopy (ie, focused on comorbidities) and comparisons among all bowel cleanser kits are generally not made. In a systematic review focusing on colonoscopy patients, the US Multi-Society Task Force guidelines on bowel cleansers failed to address any safety outcomes. ${ }^{3}$ Systematic reviews by Belsey et $a t^{10}$ and Clark et at have found adverse events associated with polyethylene glycol (PEG) and $\mathrm{NaP}$. However, we are not aware of any systematic review that has included a network meta-analysis (NMA) comparing the relative safety of all bowel cleanser products. NMA has become a vital and broadly used approach to evidence synthesis in addressing research questions that involve the comparison of multiple therapies and wherein both direct and indirect data of relevance exist $^{11-13}$; such an analysis in this area will be informative for decision making.

The current review will be performed to assess the safety concerns, specifically in relation to: (A) the comparative safety of PEG 3350-based bowel cleansers (eg, PegLyte and Bi-PegLyte) when combined with any dose of bisacodyl as a stimulant laxative; (B) the comparative safety of sodium picosulfate-based bowel cleansers when combined with any dose of bisacodyl as a stimulant laxative; and (C) the comparative safety of bowel cleansing products currently used in Canada for the purposes of preparation for colonoscopy. A preliminary scoping review (stage 1) will be conducted to map all bowel cleanser trials in the literature, their interventions and comparators and their reported safety outcomes to better inform a systematic review incorporating meta-analyses and NMAs proposed in stage 2.

\section{METHODS AND ANALYSIS}

The reporting of this protocol adheres to the Preferred Reporting Items for Systematic Review and Meta-Analysis Protocols statement. ${ }^{14}$ The checklist for reporting items can be found in the supplementary material provided. Any subsequent amendments to the protocol will be clarified in the final manuscript. The review was also registered in PROSPERO\#: CRD42018084720.

Given the vast amount of literature on bowel cleansers, the numerous products (eg, PEG 3350, PEG-ELS, sodium picosulfate and $\mathrm{NaP}$ ), the various formulations, combinations of ingredients, dosing (eg, 2L PEG solutions needing adjunct laxatives vs 4L PEG solutions alone, with or without bisacodyl tablets $<10 \mathrm{mg}$ or $\geq 10 \mathrm{mg}$, split dose vs single dose for 4L PEG solutions) and the diverse safety endpoints that could be potentially reported, the planned review will be performed in two stages (see figure 1).

\section{Stage 1}

We will conduct a scoping review of the literature to identify relevant RCTs, quasi- RCTs and large ( $>500$ patients per treatment arm) comparative, non-randomised cohort studies reporting safety endpoints of interest. A detailed summary of treatment comparisons made within these studies as well as endpoints reported will be prepared; discussion of this information within the research team will inform decisions regarding data analyses in stage 2 . The comparisons of interest that are feasible for pairwise meta-analyses and NMAs will be established based on the evidence available.

\section{Stage 2}

Pairwise meta-analyses and, where possible, NMAs will be performed to quantitatively compare safety endpoints between competing bowel cleansers.

\section{Data sources and search strategy}

Search strategies have been developed and tested through an iterative process by an experienced medical information specialist in consultation with the review team. The strategies will be peer reviewed by another senior information specialist using the Peer Review of Electronic Search Strategies (PRESS) Checklist prior to beginning the review. ${ }^{15}$ Using the OVID platform, we will search Ovid MEDLINE, including Epub Ahead of Print and In-Process \& Other Non-Indexed Citations, and Embase Classic+Embase. We will also search the Cochrane Library on Wiley.

Strategies have been designed to use a combination of controlled vocabulary (eg, 'Cathartics', 'Colonoscopy' and 'Polyethylene Glycols') and keywords (eg, 'bowel cleansers', 'colon evacuation', 'PEG' and 'purgative'). We will perform a search for primary studies using filters for randomised controlled trials (RCTs) and non-RCTs and observational studies. Vocabulary and syntax will be adjusted across databases. When possible, animal-only and opinion pieces will be removed from the results. Specific details regarding the strategies appear in the supplementary material.

A grey literature search of targeted clinical trial registries, ClinicalTrials.gov and the International Clinical Trials Registry Platform, will be performed along with searching the Canadian Agency of Drugs and Technologies in Health Grey Matters Checklist. The totality of these supplemental searches will be confined to what can be accomplished within 40 hours of work by one team member. The search details will be documented, and potentially relevant studies identified from the 
Flow Diagram of Staged Approach
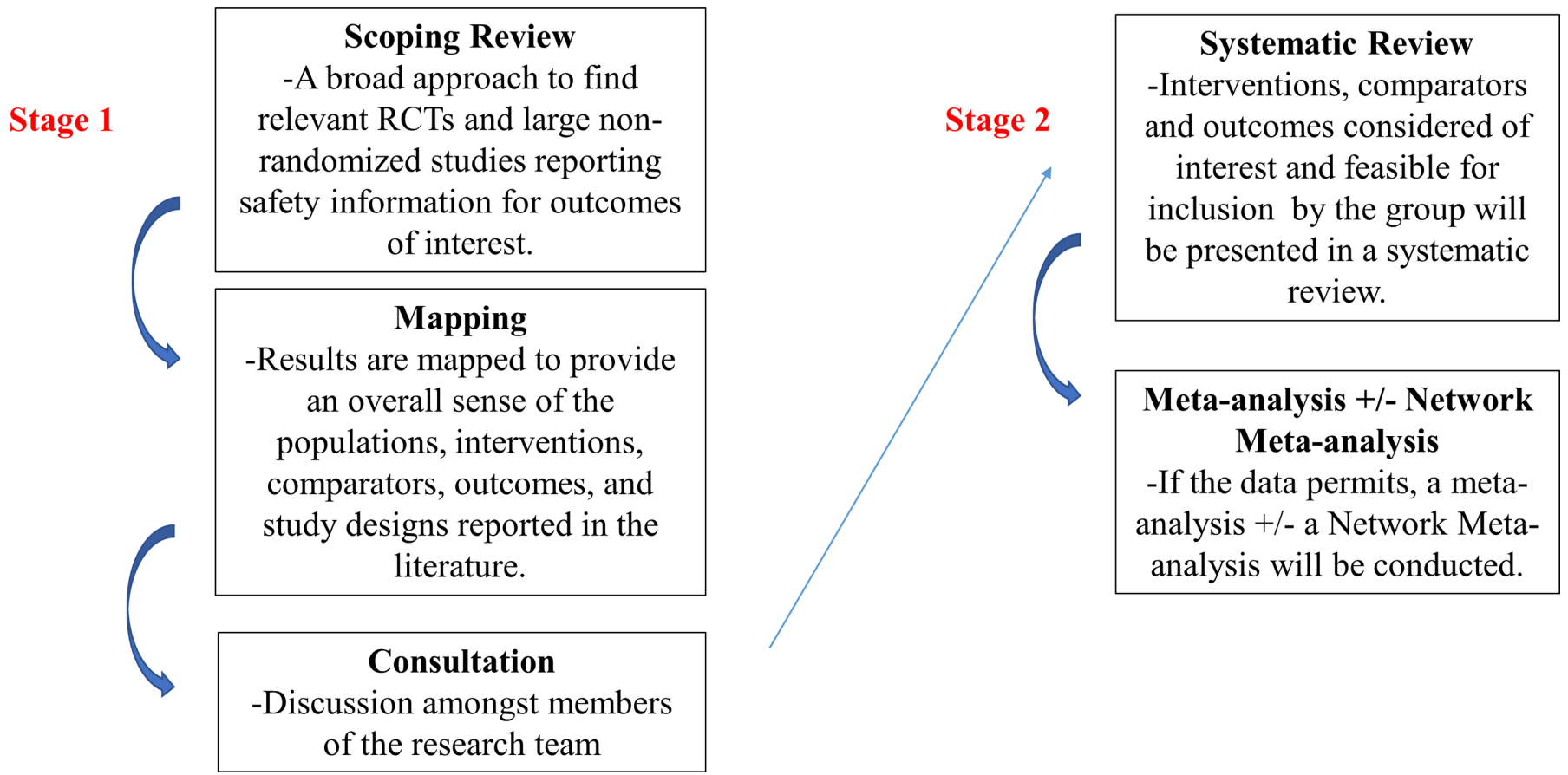

Figure 1 A flow diagram outlining the proposed stages for the scoping review and subsequent systematic review and possible meta-analysis and network meta-analysis. RCTs, randomised controlled trials.

supplemental searches will be integrated into the Preferred Reporting Items for Systematic Reviews and Meta-Analyses (PRISMA) flow diagram.

\section{Study eligibility criteria}

Population

For the main analysis, only generally healthy patients undergoing colonoscopy will be included. Subgroup populations of interest include paediatrics $(\leq 18$ years old), the elderly ( $\geq 65$ years old) and patients with comorbidities, such as inflammatory bowel disease (IBD), irritable bowel syndrome (IBS), colon cancer, diabetes, heart disease and diverticulitis. The percentage of patients in these subgroup populations as well as their related outcome data will be gathered (if available) to inform potential subgroup analyses and/or empirical evaluations of study homogeneity.

\section{Interventions and comparators of interest}

Included interventions of interest consist of (1) PEG, with or without bisacodyl; (2) sodium picosulfate, with or without bisacodyl; and (3) other types of bowel cleansers, with or without bisacodyl (eg, NaPs) (table 1). Doses of regimen components will be collected to enable stratification (eg, bisacodyl doses below or equal to vs above $10 \mathrm{mg}$ ), as well as formulation (eg, PEG $2 \mathrm{~L}$ vs $4 \mathrm{~L}$ ). Data regarding the use of boosters (eg, magnesium oxide, magnesium citrate and ascorbic acid) will also be collected. All formulations will be considered for stage 1 ; however, only those formulations available in Canada will be considered in stage 2 (table 1). Figure 2 presents preliminary network diagrams illustrating how data analyses may be structured in different ways to assess the influence of bisacodyl dosing. These structures will be discussed further among the research team after completion of the scoping review component. The comparators of interest will also include placebo or 'standard care', which could consist of another bowel cleanser; both may serve as important links/sources of indirect evidence for NMA.

\section{Outcomes}

Outcomes of interest will include the following: (1) ischaemic colitis; (2) electrolyte/fluid disturbances (eg, hyponatraemia, hypokalaemia, hypocalcaemia, hypochloraemia and dehydration) and their consequences (eg, electrocardiogram (EKG or ECG) abnormalities); (3) seizures; (4) bowel perforation; (5) hospitalisation; and (6) patient tolerability (eg, bloating, nausea/vomiting, inability to complete and acceptability score).

\section{Study designs}

Study designs of interest will include: (1) randomised controlled trials and quasirandomised trials and (2) non-randomised studies (ie, cohort studies) with suitable controls of a minimum sample size of 500 per treatment arm. Only those published in English and French will be included. Case-control studies will be excluded.

\section{Screening and data extraction}

Citations obtained from the literature search will be screened by two reviewers based on title, keywords and abstract (level 1 screening) and full-text articles (level 


\begin{tabular}{|c|c|c|}
\hline & Brand names & Status in Canada \\
\hline \multirow{5}{*}{$\begin{array}{l}\text { PEG-ELS }(2 \mathrm{~L} \text { or } 4 \mathrm{~L}) \text { : a non-absorbable polymer of } \\
\text { ethylene oxide of high molecular weight (commonly } \\
3350 \mathrm{Da}) \text {, administered in a dilute electrolyte } \\
\text { lavage solution that may or may not contain sodium } \\
\text { sulfate. The proposed mechanism is that the osmotic } \\
\text { effect of the polymer acts to retain the electrolyte } \\
\text { solution in the colon, which then acts as a bowel } \\
\text { cleanser. The sodium sulfate also has a laxative } \\
\text { effect, although its sulfur component and associated } \\
\text { rotten egg smell may reduce tolerability. }{ }^{28} 9\end{array}$} & $\begin{array}{l}\text { Moviprep ( } 2 \text { L): (PEG ELS }+10.6 \mathrm{~g} \text { of ascorbic acid/ } \\
\text { sodium ascorbate). }\end{array}$ & Currently marketed and approved in Canada. \\
\hline & $\begin{array}{l}\text { Bi-Peglyte (2 L): } \\
\text { (PEG ELS + } 15 \text { mg bisacodyl). }\end{array}$ & Currently marketed and approved in Canada. \\
\hline & GoLYTELY and CoLyte (4 L). & Currently marketed and approved in Canada. \\
\hline & $\begin{array}{l}\text { HalfLytely (2 L): (PEG-ELS without sodium } \\
\text { sulfate + bisacodyl (now reduced to } 5 \mathrm{mg} \text { due to } \\
\text { ischaemic colitis). }{ }^{28}\end{array}$ & \\
\hline & Klean-Prep (4 L). & No longer manufactured. \\
\hline \multirow{2}{*}{$\begin{array}{l}\text { Sodium picosulfate: an inactive compound that is } \\
\text { activated by the bacteria in the colon. The active } \\
\text { form of } 4,4^{\prime} \text {-dihydroxy-diphenyl-[2-pyridyl]methane } \\
\text { functions as the stimulant laxative. }{ }^{28} \text { This compound } \\
\text { is usually given in combination with an osmotic } \\
\text { laxative, such as magnesium citrate. }\end{array}$} & $\begin{array}{l}\text { Pico-Salax: } \\
\text { (sodium picosulfate + magnesium citrate). }\end{array}$ & Currently marketed and approved in Canada. \\
\hline & $\begin{array}{l}\text { Purg-Odan: } \\
\text { (sodium picosulfate + magnesium citrate). }\end{array}$ & Currently marketed and approved in Canada. \\
\hline $\begin{array}{l}\text { Sodium phosphate: } \\
\text { was once popular but no longer recommended by } \\
\text { Health Canada as a bowel cleanser due to increases } \\
\text { in reported kidney injuries. }{ }^{30} \text { Due to its significant } \\
\text { presence in the literature, it will be included as it may } \\
\text { represent a valuable link for indirect comparisons in } \\
\text { network meta-analyses. }\end{array}$ & & \\
\hline
\end{tabular}

Canadian approval status available from: https://health-products.canada.ca/dpd-bdpp/

2 screening). Level 1 will be performed using a liberal accelerated approach (ie, only one reviewer will be needed to include a citation, while two reviewers will be needed to exclude). Level 1 citations deemed potentially relevant or lacking sufficient information to decide will be carried forward to level 2, which will be performed by two reviewers independently (with disagreements settled by discussion). Where consensus is not achieved following discussion, a third independent team member will be consulted. Study screening will be conducted using Distiller Systematic Review Software (Evidence Partners; Ottawa, Canada). At both levels of screening, a pilot exercise of a small number of abstracts/full texts will be performed to establish a baseline among the reviewers to ensure consistency. The process of literature selection will be reported using a flow diagram as recommended by the PRISMA statement. ${ }^{16} 17$

Studies associated with multiple publications (eg, updates of different follow-up durations) will have the most up-to-date report retained, and a note will be made of all related manuscripts.

The screening results from the stage 1 scoping review will be the basis of the stage 2 systematic review and meta-analyses. Primary data collection of included studies will be performed independently by two reviewers using a standardised electronic data collection form in Microsoft Excel. Data gathered during stage 1 will include the patient population/indication for use of bowel cleansers, intervention and comparator information, outcomes reported and cited study design. These data will be used to develop: (1) a map of all treatment comparisons, (2) a map of all study populations/eligibility criteria, (3) a map of the outcomes reported and (4) a listing of study designs and sample sizes used. Together, these pieces of information will inform a discussion among the review team members of the network geometries feasible for analyses in stage 2 of the review.

For studies that will contribute to meta-analyses in stage 2 as established by the team's discussion of stage 1 findings, the following additional data elements will be collected: study characteristics (authors, year of publication, journal and countries of performance), patient characteristics (eligibility criteria, number per group and key demographics, including age, gender, race, comorbidities and other information as needed), interventions (regimens used as well as dosages and method of administration) and outcomes (as specified earlier, with numbers of events and numbers of patients per group for binary endpoints, which will be the format of all outcomes of interest for this review-continuous endpoints such as serum electrolyte values will not be extracted; only numbers of patients with electrolyte abnormalities). All study characteristics will be summarised in tabular form to facilitate inspection and discussion with clinical experts in terms of study heterogeneity, grouping of interventions and other such topics required to inform analysis; these tables will also be included in the final report. Collected data will be verified by a second reviewer for accuracy, with disagreements being settled by discussion.

\section{Risk of bias (RoB) assessments of included studies}

RoB will be assessed for RCTs using the Cochrane RoB tool. The Cochrane RoB tool evaluates seven domains 


\section{A}

PEG 2L+ PEG 2L+

BIS $<10 \mathrm{mg}$
BIS $>10 \mathrm{mg}$

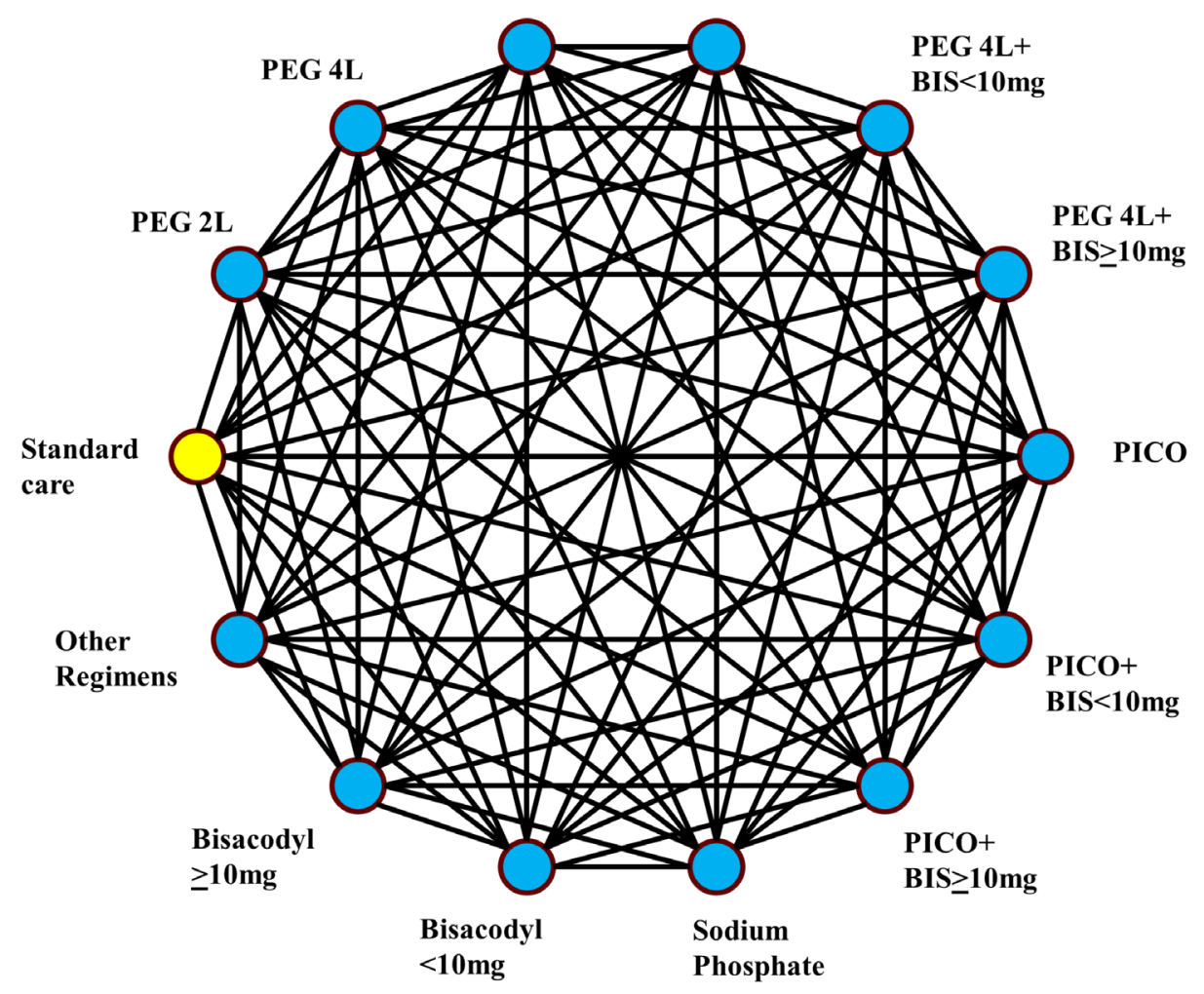

B

C

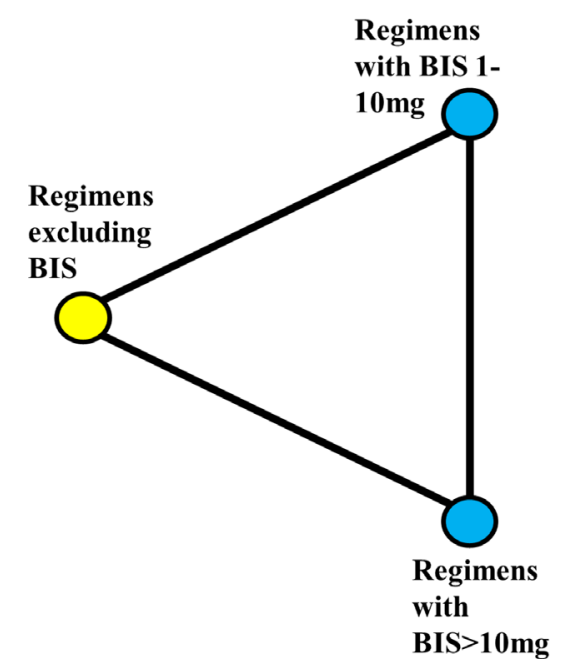

Regimens Regimens without BIS with BIS

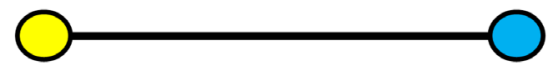

Figure 2 A preliminary schematic of different strategies to analyse the collected data to compare bowel cleanser regimens is shown. The degree to which comparisons are informed by data will be reliant upon findings from screening of the citations identified during the electronic literature search. BIS, bisacodyl; PEG, polyethylene glycol; PICO, sodium picosulfate.

including random sequence generation, allocation concealment, blinding, missing outcome data, selective outcome reporting, attrition and 'other sources of bias'. ${ }^{18}$ The quasirandomised trials will be assessed using the Risk of Bias in Non-Randomized studies of Interventions tool, ${ }^{19}$ and the non-randomised studies (cohort studies) will be assessed using the Scottish Intercollegiate
Guidelines Network 50 tool. $^{20}$ Assessments will be conducted independently by two reviewers, and any disagreements will be resolved through discussion or by third-party adjudication. Results from these appraisals will be summarised in the review and provided in full in an appendix. They will also be considered as criteria for sensitivity analyses. 


\section{Approach to evidence synthesis}

No formal quantitative analyses will be conducted in stage 1. Tabular and/or graphical summaries will be developed, presenting a comprehensive map of the populations studied, the treatment comparisons assessed and the outcomes of interest. This information will directly inform decisions regarding the analyses to be undertaken in stage 2. In stage 2 , for the outcomes found to have sufficient data for analysis, we will first conduct traditional meta-analyses of all pairwise comparisons in the evidence networks. NMAs will subsequently be performed, if there is sufficient homogeneity. Primary analyses for all outcomes will first be based on data from RCTs only. Subsequently, incorporation of data from non-randomised sources will be undertaken sequentially, allowing transparent assessment of the influence of the non-randomised information on overall findings.

An initial exploration of potential clinical and methodological heterogeneity among the included studies will be conducted using tabular and graphical approaches. The assumption of transitivity will be evaluated by inspection of differences in patient eligibility criteria and pertinent patient demographics between studies, including comorbidities (eg, presence of IBS, IBD, heart disease and diverticulitis) and age (eg, paediatric and elderly patients). Methodological differences between studies that could influence outcome measurements will be noted. We will describe any concerns related to the transitivity assumption or methodological heterogeneity within the final report and consider statistical strategies to account for address any concerns.

Standard pairwise meta-analyses will be conducted by fitting random-effects models in Comprehensive Meta-Analysis V.3 software (Biostat; Englewood, New Jersey, USA) to generate summary estimates and to assess statistical heterogeneity across the included studies. All summary estimates will be reported as ORs, with corresponding 95\% CIs. Statistical heterogeneity will be measured by the $\mathrm{I}^{2}$ statistic. $\mathrm{I}^{2}$ values of $50 \%$ or higher will be considered indicative of potentially important heterogeneity that will be explored using established methods such as subgroup analysis, meta-regression and/or exclusion of outlier studies. If necessary, similar approaches will be conducted in NMAs to address existing heterogeneity.

Where feasible, NMAs will be carried out separately for each clinical outcome of interest. Approaches used for these analyses will follow existing recommendations for modelling of unadjusted and adjusted models as outlined by guidance from experts at the National Institute for Health and Care Excellence. ${ }^{21-23}$ Both fixed-effect and random-effects models will be fit for each outcome, within a Bayesian framework. Totals of 50000 or more burn-in iterations and 50000 or more sampling iterations will be used for all NMAs, and model convergence will be assessed based on inspection of history plots and the Monte Carlo error of all parameters. The fit of a model will be assessed by comparing its posterior residual deviance with the number of unconstrained data points (ie, the number of intervention arms across all studies) for the analysis. Selection between different models will be based on deviance information criteria (DIC) for each competing model, with a difference of 5 or more points to be considered significant. For networks where statistical heterogeneity is high or the number of single-study connections is high, random-effects models will be preferred. Summary ORs will be estimated for all possible pairwise comparisons in a network. The assumption of consistency of direct and indirect evidence will be assessed by comparison of ORs from traditional pairwise meta-analyses with those estimated in the NMA. Consistency will be assessed further by (1) fitting inconsistency NMA models and comparing the DIC with the corresponding consistency models; and (2) reviewing scatterplots of the residuals from these models. Probabilities of treatment superiority will be estimated using the Surface Under the Cumulative Ranking curve (SUCRA), and ranking of treatments will be estimated by median treatment rankings, with corresponding $95 \%$ credible intervals. All NMAs will be performed using WinBUGS software V.1.4.3 (MRC Biostatistics Unit), with the Microsoft Excel plug-in tool NetMetaXL to organise datasets and generate summary figures, including network diagrams, forest plots, league tables of SUCRA values and rankograms of median treatment rankings. ${ }^{24}$

\section{Subgroup and sensitivity analyses}

Primary analyses will be unadjusted; however, additional analyses to assess heterogeneity will be pursued if feasible based on data availability and network geometry considerations (eg, few single-study connections). We will consider additional analyses to address study deficiencies found in risk-of-bias assessments by excluding low-quality studies. Meta-regression and/or subgroup meta-analyses will also be pursued to address clinically important variations between studies with regard to patient characteristics, including patient age (ie, paediatrics ( $\leq 18$ years) and the elderly ( $\geq 65$ years) ) and prevalence of comorbidities (eg, IBD, IBS, colon cancer, diabetes, heart failure, renal failure and diverticulitis). Based on previous literature and clinical expert input, we anticipate few studies will provide outcome data specific to these groups, and thus sensitivity analyses including (and excluding) studies with high proportions of the different subgroups may be the only feasible approach to assess their effect on overall review findings.

\section{Reporting of review findings}

Full graphical and numeric presentations of findings will be prepared. This will include the following for each feasible outcome of interest: network diagrams showing the availability of evidence for all possible treatment comparisons; summary ORs, with $95 \%$ credible intervals for all pairwise comparisons in the network; SUCRA values and median treatment rankings, with corresponding $95 \%$ credible interval for all treatments in the network. These will be described using approaches recommended by Salanti et al. ${ }^{25}$ We will use the checklist of the PRISMA 
Statement for Network Meta-Analysis to ensure all findings are clearly reported. ${ }^{17}$ If NMA is not feasible, pairwise meta-analysis findings will be reported in tables with a narrative summary.

\section{Patient and public involvement}

Patients were not directly consulted during the planning of this research. Following completion of this work, we will disseminate our findings through open-access publications as well as the preparation of lay summaries to be made available through our funder's website.

\section{ETHICS AND DISSEMINATION}

Due to the nature of the study design being employed (scoping review followed by systematic review with possible NMA), ethics approval will not need to be sought.

In the USA, the FDA has withdrawn from market the bowel cleanser kit HalfLytely with $10 \mathrm{mg}$ bisacodyl tablets due to concerns regarding higher incidence of ischaemic colitis relative to kits with $5 \mathrm{mg}$ bisacodyl. Currently in Canada, a bowel cleanser kit with $15 \mathrm{mg}$ bisacodyl is approved and marketed. The review of the comparative safety of approved bowel cleansers for healthy patients undergoing colonoscopy in Canada will inform policy and regulatory guidelines.

Although NMAs have been published evaluating bowel cleanser efficacy, they have focused on different patient indications (such as colorectal surgery and constipation) ${ }^{2627}$ and they often do not evaluate safety outcomes. ${ }^{3}$ To our knowledge, this would be the first NMA focused on bowel cleanser safety in healthy colonoscopy patients.

We anticipate certain challenges in the context of the current review. Patient comorbidities (eg, IBS) are known to influence the risk of certain endpoints such as ischaemic colitis. Evaluation of the comparative frequencies of harms between comorbidity subgroups will not be possible if insufficient subgroup data are available, preventing quantitative assessment of the potential influence of varying patient comorbidities. The synthesis of combined data from randomised and non-randomised studies remains an active area of research. We will employ a sequential approach to our meta-analyses, such that the impact of data from non-randomised studies on summary findings will be transparent to readers. On completion, results will be submitted for publication in a peer-reviewed journal.

Acknowledgements We would like to thank Raymond Daniel (assistant information specialist) for his role in providing support in database management and document procurement.

Contributors $\mathrm{PB}, \mathrm{BH}$ and DW drafted the protocol. BS created and tested the search strategies to be used in the bibliographic databases. SK, CD, AR, DM and $\mathrm{BH}$ provided clinical expertise during the protocol development stage and will also provide such expertise throughout the duration of the review and during drafting of the final manuscript. PB, DW, FY and DBR will be involved in screening, data extraction and quality assessment. $\mathrm{PB}, \mathrm{DW}$ and $\mathrm{BH}$ will be involved in all data analyses. PB, DW, and BH will coordinate all aspects of the review. BH conceived of and is the guarantor of the review. All authors reviewed and approved the protocol.
Funding This work was supported by the Canadian Institutes of Health Research's Drug Safety and Effectiveness Network. DBR is supported by a Vanier Graduate Scholarship.

Disclaimer The funders had no role in the design of the planned study or preparation of this protocol.

Competing interests $\mathrm{BH}$ has previously received honoraria from Cornerstone Research Group for the provision of methodological expertise related to the conduct of systematic reviews and meta-analyses.

Patient consent Not required.

Provenance and peer review Not commissioned; externally peer reviewed.

Open access This is an open access article distributed in accordance with the Creative Commons Attribution Non Commercial (CC BY-NC 4.0) license, which permits others to distribute, remix, adapt, build upon this work non-commercially, and license their derivative works on different terms, provided the original work is properly cited and the use is non-commercial. See: http://creativecommons.org/ licenses/by-nc/4.0/

(c) Article author(s) (or their employer(s) unless otherwise stated in the text of the article) 2018. All rights reserved. No commercial use is permitted unless otherwise expressly granted.

\section{REFERENCES}

1. Harrison NM, Hjelkrem MC. Bowel cleansing before colonoscopy: Balancing efficacy, safety, cost and patient tolerance. World $\mathrm{J}$ Gastrointest Endosc 2016;8:4-12.

2. Summers R. The Elephant in the Room. Acad Radiol 2009;16:777-9.

3. Johnson DA, Barkun AN, Cohen LB, et al. US Multi-Society Task Force on Colorectal Cancer. Optimizing adequacy of bowel cleansing for colonoscopy: recommendations from the US multi-society task force on colorectal cancer. Gastroenterology 2014;147:903-24.

4. Bechtold ML, Mir F, Puli SR, et al. Optimizing bowel preparation for colonoscopy: a guide to enhance quality of visualization. Ann Gastroenterol 2016;29:137-46.

5. Food and Drug Administration. Detertmination that Halflytely and Bisacodyl tablets bowel prep kit (containing two bisacodyl delayed release tablets, 5 milligrams) was withdrawn from sale for reasons of safety or effectiveness [Internet]. https://www.federalregister.gov/ documents/2011/08/17/2011-20853/determination-that-halflytelyand-bisacodyl-tablets-bowel-prep-kit-containing-two-bisacodyldelayed (accessed 30 on Dec 2017).

6. Clark RE, Godfrey JD, Choudhary A, et al. Low-volume polyethylene glycol and bisacodyl for bowel preparation prior to colonoscopy: a meta-analysis. Ann Gastroenterol 2013;26:319-24.

7. Enestvedt BK, Tofani C, Laine LA, et al. 4-Liter split-dose polyethylene glycol is superior to other bowel preparations, based on systematic review and meta-analysis. Clin Gastroenterol Hepatol 2012;10:1225-31.

8. Juluri R, Eckert G, Imperiale TF. Meta-analysis: randomized controlled trials of 4-L polyethylene glycol and sodium phosphate solution as bowel preparation for colonoscopy: Meta-analysis: PEG vs. NaP for bowel preparation. Aliment Pharmacol Ther 2010;32:171-81.

9. Belsey J, Epstein O, Heresbach D. Systematic review: oral bowel preparation for colonoscopy: Systematic Review: oral bowel preparation for colonoscopy. Aliment Pharmacol Ther 2006;25:373-84.

10. Belsey J, Epstein O, Heresbach D. Systematic review: adverse event reports for oral sodium phosphate and polyethylene glycol. Aliment Pharmacol Ther 2009;29:15-28.

11. Catalá-López F, Tobías A, Cameron C, et al. Network meta-analysis for comparing treatment effects of multiple interventions: an introduction. Rheumatol Int 2014;34:1489-96.

12. Caldwell DM, Ades AE, Higgins JP. Simultaneous comparison of multiple treatments: combining direct and indirect evidence. BMJ 2005;331:897-900.

13. Salanti G. Indirect and mixed-treatment comparison, network, or multiple-treatments meta-analysis: many names, many benefits, many concerns for the next generation evidence synthesis tool. Res Synth Methods 2012;3:80-97.

14. Moher D, Shamseer L, Clarke M, et al. Preferred reporting items for systematic review and meta-analysis protocols (PRISMA-P) 2015 statement. Syst Rev 2015;4:1. 
15. McGowan J, Sampson M, Salzwedel DM, et al. PRESS Peer Review of Electronic Search Strategies: 2015 Guideline Statement. J Clin Epidemiol 2016;75:40-6.

16. Moher D, Liberati A, Tetzlaff J, et al. Preferred reporting items for systematic reviews and meta-analyses: the PRISMA statement. PLoS Med 2008:6.

17. Hutton B, Salanti G, Caldwell DM, et al. The PRISMA extension statement for reporting of systematic reviews incorporating network meta-analyses of health care interventions: checklist and explanations. Ann Intern Med 2015;162:777-84.

18. Higgins JP, Altman DG, Gøtzsche PC, et al. The Cochrane Collaboration's tool for assessing risk of bias in randomised trials. BMJ 2011:343:d5928.

19. Sterne JA, Hernán MA, Reeves BC, et al. ROBINS-I: a tool for assessing risk of bias in non-randomised studies of interventions. BMJ 2016;355:i4919.

20. SIGN. SIGN-Critical Appraisal Notes and Checklists- Methodology Checklist 3: Cohort studies [Internet]. http://www.sign.ac.uk/ checklists-and-notes.html (accessed 30 Dec 2017).

21. Dias S, Welton N, Sutton A, et al. NICE DSU Technical Support Document 2: A Generalised Linear Modelling Framework for Pairwise and Network Meta-Analysis of Randomised Controlled Trials[Internet]. $2011 \mathrm{http} / / /$ www.nicedsu.org.uk/TSD2\%20General\% 20meta\%20analysis\%20corrected\%202Sep2016v2.pdf (accessed 30 Dec 2017).

22. Dias S, Sutton A, Welton N, et al. NICE DSU Technical Support Document 3: Heterogeneity: subgroups, meta-regression, bias and bias-adjustment [Internet]. $2012 \mathrm{http}: / /$ www.nicedsu.org.uk/TSD3\% 20Heterogeneity.final\%20report.08.05.12.pdf (accessed 30 Dec 2017).
23. Dias $\mathrm{S}$, Welton NJ, Sutton AJ, et al. Evidence synthesis for decision making 4: inconsistency in networks of evidence based on randomized controlled trials. Med Decis Making 2013;33:641-56.

24. Brown S, Hutton B, Clifford T, et al. A Microsoft-Excel-based tool for running and critically appraising network meta-analyses--an overview and application of NetMetaXL. Syst Rev 2014;3:110.

25. Salanti G, Ades AE, loannidis JP. Graphical methods and numerical summaries for presenting results from multipletreatment meta-analysis: an overview and tutorial. $J$ Clin Epidemiol 2011:64:163-71.

26. Dahabreh IJ, Steele DW, Shah N, et al. Oral Mechanical Bowel Preparation for Colorectal surgery- Effective Health Care Program. Maryland, USA: Agency for Healthcare Research and Quality, 2014. Comparative Effectiveness Review. No. 128.

27. Katelaris P, Naganathan V, Liu K, et al. Comparison of the effectiveness of polyethylene glycol with and without electrolytes in constipation: a systematic review and network meta-analysis. BMC Gastroenterol 2016;16:42.

28. Adamcewicz M, Bearelly D, Porat G, et al. Mechanism of action and toxicities of purgatives used for colonoscopy preparation. Expert Opin Drug Metab Toxicol 2011;7:89-101.

29. Schanz S, Kruis W, Mickisch O, et al. Bowel Preparation for Colonoscopy with Sodium Phosphate Solution versus Polyethylene Glycol-Based Lavage: A Multicenter Trial. Diagn Ther Endosc 2008;2008:1-6.

30. Health Canada. Health Canada warns that use of oral sodium phosphate products for bowel cleansing may lead to kidney injury [Internet]Mar. 2009 http://healthycanadians.gc.ca/recall-alertrappel-avis/hc-sc/2009/13368a-eng.php (accessed on 30 Dec 2017). 